\title{
Remarks on sum rules in the heavy quark limit of QCD
}

\section{A. Le Yaouanc, L. Oliver*, O. Pène and J.-C. Raynal}

Laboratoire de Physique Théorique (UMR CNRS 8627), Université de Paris-Sud, bâtiment 210, 91405 Orsay Cedex, France

\section{Morénas}

Laboratoire de Physique Corpusculaire, Université Blaise Pascal, CNRS/IN2P3, 63000 Aubière Cedex, France

ABstract: We underline a problem existing in the heavy quark limit of QCD concerning the rates of semileptonic $B$ decays into $P$-wave $D_{J}(j)$ mesons, where $j=\frac{1}{2}$ (wide states) or $j=\frac{3}{2}$ (narrow states). The leading order sum rules of Bjorken and Uraltsev suggest $\Gamma\left[\bar{B} \rightarrow D_{0,1}\left(\frac{1}{2}\right) \ell \nu\right] \ll \Gamma\left[\bar{B} \rightarrow D_{1,2}\left(\frac{3}{2}\right) \ell \nu\right]$, in contradiction with experiment. The same trend follows also from a sum rule for the subleading $1 / m_{Q}$ current matrix element correction $\xi_{3}(1)$. The problem is made explicit in relativistic quarks models à la Bakamjian and Thomas, that give a transparent physical interpretation of the latter as due, not to a $\mathbf{L} \cdot \mathbf{S}$ force, but to the Wigner rotation of the light quark spin. We point out moreover that the selection rule for decay constants of $j=\frac{3}{2}$ states, $f_{3 / 2}=0$, predicts, assuming the model of factorization, the opposite hierarchy $\Gamma\left[\bar{B} \rightarrow \bar{D}_{s_{1,2}}\left(\frac{3}{2}\right) D^{(*)}\right] \ll \Gamma\left[\bar{B} \rightarrow \bar{D}_{s_{0,1}}\left(\frac{1}{2}\right) D^{(*)}\right]$.

It has been recently pointed out by Uraltsev iij that the function $T\left(\varepsilon, \mathbf{v}, \mathbf{v}-\mathbf{v}^{\prime}\right)$, Fourier transform of $<B^{*}\left(\mathbf{v}-\mathbf{v}^{\prime}\right)\left|T\left(J^{+}(0) J(x)\right)\right| B^{*}(0)>$ (where the momentum transfer is $\left.-m_{Q} \mathbf{v}\right)$, can be decomposed into symmetric and antisymmetric parts in $\mathbf{v}, \mathbf{v}^{\prime}, h_{ \pm}(\varepsilon)$.

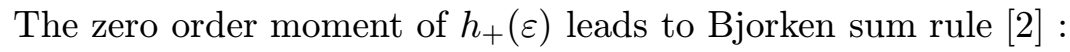

$$
\rho^{2}=\frac{1}{4}+\sum_{n}\left|\tau_{1 / 2}^{(n)}(1)\right|^{2}+2 \sum_{n}\left|\tau_{3 / 2}^{(n)}(1)\right|^{2}
$$

while the zero order moment of $h_{-}(\varepsilon)$ gives a new sum rule :

$$
\sum_{n}\left|\tau_{3 / 2}^{(n)}(1)\right|^{2}-\sum_{n}\left|\tau_{1 / 2}^{(n)}(1)\right|^{2}=\frac{1}{4}
$$

Note that this relation is to be contrasted with the non-relativistic quark model with spinorbit independent interactions [i3i] : $\tau_{3 / 2}^{(n)}(1)=\tau_{1 / 2}^{(n)}(1)$. Indeed, Uraltsev SR corresponds to subleading corrections in the non-relativistic expansion. In these relations $\tau_{j}(w)$ are the two

${ }^{*}$ Speaker. 
independent IW functions for the $B \rightarrow D_{J}(j)$ transitions $\left(J=0,1\right.$, for $j=\frac{1}{2}$ and $J=1,2$ for $j=\frac{3}{2}$ ). From both sum rules it follows the lower bound for the elastic IW function $\rho^{2} \geq \frac{3}{4}$. This bound was obtained some years ago within Bakamjian-Thomas quark models

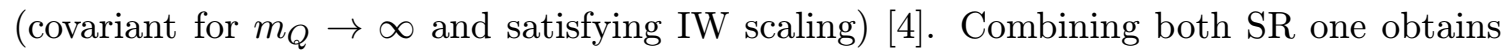
$\sum_{n}\left|\tau_{1 / 2}^{(n)}(1)\right|^{2}=\frac{1}{3}\left(\rho^{2}-\frac{3}{4}\right)$. This simple relation shows that, since $\rho^{2}$ is of $O(1)$, there is little room left for the sum $\sum_{n}\left|\tau_{1 / 2}^{(n)}(1)\right|^{2}$, compared to $\sum_{n}\left|\tau_{3 / 2}^{(n)}(1)\right|^{2}$. The same qualitative

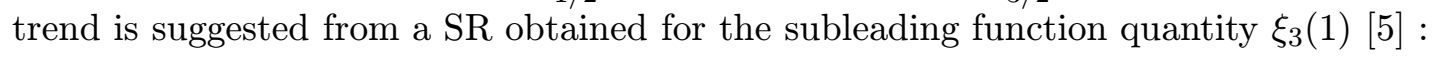

$$
\xi_{3}(1)=2 \sum_{n} \Delta E_{3 / 2}^{(n)}\left|\tau_{3 / 2}^{(n)}(1)\right|^{2}-2 \sum_{n} \Delta E_{1 / 2}^{(n)}\left|\tau_{1 / 2}^{(n)}(1)\right|^{2}
$$

$\xi_{3}(1)$ and $\bar{\Lambda}=m_{B}-m_{b}$ describe, at zero recoil, the subleading corrections to the ground state current matrix elements due to the perturbations of the current, the quantities denoted by $L_{i}(1)(i=4,5,6)$ in the notation of Falk and Neubert [i-i]. Note that $\xi_{3}(1)$ is

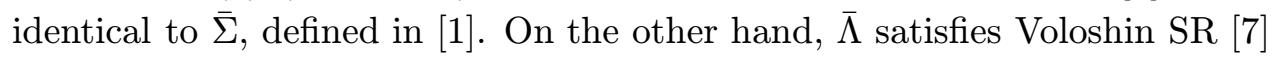

$$
\frac{\bar{\Lambda}}{2}=\sum_{n} \Delta E_{1 / 2}^{(n)}\left|\tau_{3 / 2}^{(n)}(1)\right|^{2}+2 \sum_{n} \Delta E_{3 / 2}^{(n)}\left|\tau_{3 / 2}^{(n)}(1)\right|^{2} \quad .
$$

If one combines both subleading SR together with the QCDSR result (ignoring short distance corrections) [票] $\xi_{3}(1)=\frac{\bar{\Lambda}}{3}$ one obtains the ratio $\sum_{n} \Delta E_{3 / 2}^{(n)}\left|\tau_{3 / 2}^{(n)}(1)\right|^{2} / \sum_{n} \Delta E_{1 / 2}^{(n)}\left|\tau_{1 / 2}^{(n)}(1)\right|^{2}$ $=4$. Since in quarkonia the $\mathbf{L} \cdot \mathbf{S}$ force turns out to be small, the level spacings $\Delta E_{j}^{(n)}$ are roughly independent of $j$. One concludes again that one expects the hierarchy $\sum_{n}\left|\tau_{3 / 2}^{(n)}(1)\right|^{2}$ $\gg \sum_{n}\left|\tau_{1 / 2}^{(n)}(1)\right|^{2}$. Making the natural hypothesis that this hierarchy holds for the $n=0$ states, one expects the inequalities [i9i]

$$
\begin{aligned}
& \Gamma\left[\bar{B}_{d} \rightarrow D_{1,2}\left(\frac{3}{2}\right) \ell \nu\right] \gg \Gamma\left[\bar{B}_{d} \rightarrow D_{0,1}\left(\frac{1}{2}\right) \ell \nu\right] \\
& \Gamma\left[\bar{B}_{d} \rightarrow D_{1,2}\left(\frac{3}{2}\right) \pi\right] \gg \Gamma\left[\bar{B}_{d} \rightarrow D_{0,1}\left(\frac{1}{2}\right) \pi\right]
\end{aligned}
$$

where the second inequality follows assuming factorization for $B \rightarrow D_{J}(j) \pi\left(j=\frac{1}{2}, \frac{3}{2}\right)$, a natural hypothesis taking into account the recent work in QCD on factorization in twobody non-leptonic decays when a light meson is emitted [1] $\left[\begin{array}{l}1 \\ 0\end{array}\right]$. However, experiment does

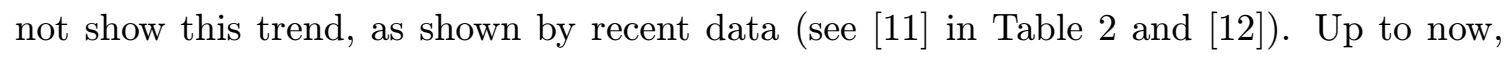
our considerations have been model-independent.

To illustrate different aspects, let us consider the Bakamjian-Thomas quark models [- These are relativistic quark models with an interacting fixed number of constituents, the states forming representations of the Poincaré Group. It has been shown that, in the heavy quark limit, current form factors are covariant, and Isgur-Wise scaling is satisfied [4] them characterized by a mass operator $M$ Ansatz, that describes the dynamics at rest. One can thus make use of the different spectroscopic models proposed in the literature and compute the elastic IW function $\xi(w)$ and the transition $P$-wave IW functions $\tau_{1 / 2}(w)$,

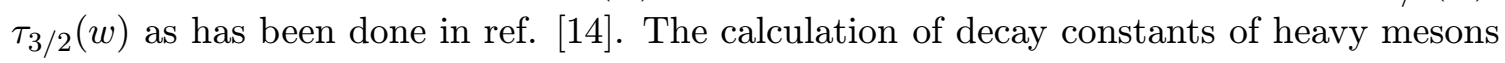


for which HQET scaling is also satisfied has been also performed within the same approach [1] that also gives a transparent physical interpretation of the difference $\tau_{3 / 2}^{(n)}(1)-\tau_{1 / 2}^{(n)}(1)$. In these classes of models one has, in terms of wave functions at rest [i [īin]

$$
\tau_{3 / 2}^{(n)}(1)-\tau_{1 / 2}^{(n)}(1) \cong \frac{1}{(2 \pi)^{2} \sqrt{3}} \int p^{2} d p\left[p \varphi_{L=1}^{(n)}(p)\right]^{*} \frac{p}{p_{0}+m} \varphi(p)
$$

where, assuming small $\mathbf{L} \cdot \mathbf{S}$ coupling, $\varphi_{1 / 2}^{(n)}(p) \cong \varphi_{3 / 2}^{(n)}(p)=\varphi_{L=1}^{(n)}(p)$, and $m, p$ and $p_{0}$ are the light quark mass, momentum and energy in the hadron rest frame. In this limit, the difference is due to the relativistic structure of the current matrix elements in terms of wave functions, namely due to the light spectator quark Wigner rotations, i.e. a relativistic kinematical effect from the center-of-mass boost. The difference is large : for a constituent quark mass $m \cong 0.3 \mathrm{GeV}, \frac{p}{p_{0}+m}$ is of $O(1)$. It must be emphasized that this effect is independent of a possible difference that could come from the small spin-orbit force that yields a difference between the internal wave functions at rest $\varphi_{1 / 2}^{(n)}(p), \varphi_{3 / 2}^{(n)}(p)$. Uraltsev SR follows in the BT scheme as follows. A change of variables is performed between the quark momenta $\left(\mathbf{p}_{1}, \mathbf{p}_{2}\right) \rightarrow\left(\mathbf{P}, \mathbf{k}_{2}\right), \mathbf{P}$ being the center-of-mass momentum, and $\mathbf{k}_{2}$ the internal relative momentum. A current matrix element writes

$$
\begin{aligned}
& <\mathbf{v}^{\prime}\left|J_{\mu}(0)\right| \mathbf{v}>=\sum_{s_{1}^{\prime} s_{1}} \bar{u}_{s_{1}^{\prime}} \gamma_{\mu} u_{s_{1}} \int d \mathbf{p}_{2} \frac{\sqrt{\left(p_{i} \cdot v\right)\left(p_{i}^{\prime} \cdot v^{\prime}\right)}}{p_{2}^{0}} \\
& \sum_{s_{2}^{\prime} s_{2}} \varphi_{s_{2}^{\prime} s_{2}}^{\prime *}\left(\mathbf{k}_{2}^{\prime}\right)\left[D\left(R_{2}^{\prime-1} R_{2}\right)\right]_{s_{2}^{\prime} s_{2}} \varphi_{s_{1} s_{2}}\left(\mathbf{k}_{2}\right)
\end{aligned}
$$

where $u_{s_{1}^{\prime}} \gamma_{\mu} u_{s_{1}}$ expresses the fact that quark 1 is the active heavy quark. The first term under the integral is the Jacobian of the change of variables. The functions $\varphi$ and $\varphi^{\prime}$ are wave functions at rest, dependent on relative momenta and Pauli spinors. The matrix $D\left(R_{2}^{\prime}-1 R_{2}\right)$ is the Wigner rotation on the spectator quark spin due to the boosts on initial

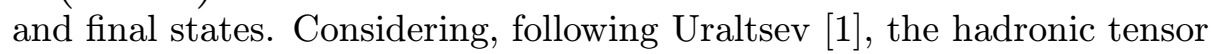

$$
h_{00}^{+10}\left(\mathbf{v}_{f}, \mathbf{v}^{\prime}, \mathbf{v}_{i}\right)=\sum_{n}<B^{*(+1)}\left(\mathbf{v}_{f}\right)\left|V_{0}(0)\right| n\left(\mathbf{v}^{\prime}\right)><n\left(\mathbf{v}^{\prime}\right)\left|V_{0}(0)\right| B^{*(0)}\left(\mathbf{v}_{i}\right)>.
$$

An expansion in the BT scheme yields

$$
<n\left(\mathbf{v}^{\prime}\right)\left|V_{0}(0)\right| O(\mathbf{v})>\cong(n \mid 0)+\frac{1}{2}\left(\mathbf{v}^{\prime}-\mathbf{v}\right) \cdot\left(n\left|-i\left(p_{2}^{0} \mathbf{r}_{2}+\mathbf{r}_{2} p_{2}^{0}\right)+\frac{i\left(\sigma_{2} \times \mathbf{p}_{2}\right)}{p_{2}^{0}+m}\right| 0\right) .
$$

Where the operator $i\left(p_{2}^{0} \mathbf{r}_{2}+\mathbf{r}_{2} p_{2}^{0}\right)$ becomes the dipole operator $2 i m \mathbf{r}_{2}$ in the non-relativistic limit and $\frac{i\left(\sigma_{2} \times \mathbf{p}_{2}\right)}{p_{2}^{0}+m}$ is a Wigner rotation. For $V_{0}$, the active quark $\bar{u}_{s_{1}^{\prime}} \gamma_{0} u_{s_{1}}$ cannot produce the necessary spin flip $B^{*(0)} \rightarrow B^{*(+1)}$, but this is produced by the Wigner rotation of the spectator light quark. One obtains

$$
h_{00}^{+10}\left(\mathbf{v}_{f}, \mathbf{v}^{\prime}, \mathbf{v}_{i}\right) \cong \frac{1}{4} v_{f}^{z} \frac{1}{\sqrt{2}}\left(v^{\prime x}-i v^{\prime y}\right)
$$

expressing quark-hadron duality. The same hadronic tensor in terms of phenomenological IW functions $\tau_{j}(w)$ yields

$$
h_{00}^{+10}\left(\mathbf{v}_{f}, \mathbf{v}^{\prime}, \mathbf{v}_{i}\right) \cong v_{f}^{z} \frac{1}{\sqrt{2}}\left(v^{\prime x}-i v^{\prime y}\right)\left[\sum_{n}\left|\tau_{3 / 2}^{(n)}(1)\right|^{2}-\sum_{n}\left|\tau_{1 / 2}^{(n)}(1)\right|^{2}\right]
$$


and hence Uraltsev SR follows. Tables 1 and 2 show calculations in the BT scheme for different dynamics at rest provided by different studies of meson spectroscopy. One can see that there is a quick convergence of Bjorken and Uraltsev sum rules, almost saturated by $n=0$ states.

\begin{tabular}{|c|c|c|c|}
\hline $\begin{array}{c}\text { Quark-antiquark } \\
\text { Potential }\end{array}$ & $\begin{array}{l}\left.\text { Godfrey, Isgur [i] }{ }^{1} \bar{k} \text { ' }\right] \\
\quad(Q \bar{Q}, Q \bar{q}, q \bar{q})\end{array}$ & $\begin{array}{c}\text { Cea, Colangelo, } \\
\left.\text { Cosmai, Nardulli [i] }{ }^{1} \overline{1} \overline{1}\right]\end{array}$ & $\begin{array}{c}\text { Isgur, Scora } \\
\text { Grinstein, Wise [1] }\left[\begin{array}{l}1 \\
1\end{array}\right]\end{array}$ \\
\hline$\left|\tau_{1 / 2}^{(0)}(1)\right|^{2}$ & 0.051 & 0.004 & 0.117 \\
\hline$\left|\tau_{3 / 2}^{(0)}(1)\right|^{2}$ & 0.291 & 0.265 & 0.305 \\
\hline$\frac{1}{4}+\left|\tau_{1 / 2}^{(0)}(1)\right|^{2}+2\left|\tau_{3 / 2}^{(0)}(1)\right|^{2}$ & 0.882 & 0.790 & 1.068 \\
\hline$\rho^{2}$ & 1.023 & 0.98 & 1.283 \\
\hline$\left|\tau_{3 / 2}^{(0)}(1)\right|^{2}-\left|\tau_{1 / 2}^{(0)}(1)\right|^{2}$ & 0.240 & 0.261 & 0.233 \\
\hline
\end{tabular}

Table 1: Contributions of the $n=0$ states to the Sum Rules in BT models

\begin{tabular}{|c|c|c|c|c|}
\hline Semileptonic Mode & Godfrey-Isgur & Cea et al. & Isgur et al. & Expt. [i] $\left.]_{1}^{1}\right]$ \\
\hline$B \rightarrow D \ell \nu$ & $2.36 \%$ & $2.45 \%$ & $1.94 \%$ & $(2.1 \pm 0.2) \%$ \\
\hline$B \rightarrow D^{*} \ell \nu$ & $6.86 \%$ & $7.02 \%$ & $6.07 \%$ & $(5.3 \pm 0.8) \%$ \\
\hline$B \rightarrow D_{2} \ell \nu$ & $7.0 \times 10^{-3}$ & $6.5 \times 10^{-3}$ & $7.7 \times 10^{-3}$ & $\begin{array}{c}\text { (a) }(2.4 \pm 1.1) \times 10^{-3} \\
\text { (b) }(4.4 \pm 2.4) \times 10^{-3} \\
(\mathrm{c})(3.0 \pm 3.4) \times 10^{-3}\end{array}$ \\
\hline$B \rightarrow D_{1}\left(\frac{3}{2}\right) \ell \nu$ & $4.5 \times 10^{-3}$ & $4.2 \times 10^{-3}$ & $4.9 \times 10^{-3}$ & $\begin{array}{c}\text { (a) }(7.0 \pm 1.6) \times 10^{-3} \\
\text { (b) }(6.7 \pm 2.1) \times 10^{-3} \\
\text { (c) }(5.6 \pm 1.6) \times 10^{-3}\end{array}$ \\
\hline$B \rightarrow D_{1}^{*}\left(\frac{1}{2}\right) \ell \nu$ & $7 \times 10^{-4}$ & $4 \times 10^{-5}$ & $1.3 \times 10^{-3}$ & $\begin{array}{c}\text { (d) }(2.3 \pm 0.7) \times 10^{-2} \\
{\left[D_{0}^{*}\left(\frac{1}{2}\right)+D_{1}^{*}\left(\frac{1}{2}\right)\right]}\end{array}$ \\
\hline$B \rightarrow D_{0}^{*}\left(\frac{1}{2}\right) \ell \nu$ & $6 \times 10^{-4}$ & $4 \times 10^{-5}$ & $1.1 \times 10^{-3}$ &
\end{tabular}

Table 2: Comparison between semileptonic decay rates in BT models and data : (a) ALEPH, (b) DELPHI, (c) CLEO [1]

Let us now comment on the decay constants of $P$-wave mesons in the heavy quark limit of QCD. In this limit, the following selection rule was found [i] $\left.\overline{1}_{-1}\right] f_{3 / 2}^{(n)}=0$. Assuming the model of factorization one finds the hierarchy in the emission of $P$-wave mesons

$$
\Gamma\left(\bar{B}_{d} \rightarrow \bar{D}_{s_{1,2}}\left(\frac{3}{2}\right) D^{(*)}\right) \ll \Gamma\left(\bar{B}_{d} \rightarrow \bar{D}_{s_{0,1}}\left(\frac{1}{2}\right) D^{(*)}\right)
$$

The BaBar experiment is looking for $\bar{B}_{d} \rightarrow(\bar{D} K) D^{(*)}$ that could allow to test this inequality. This is interesting, because it goes on exactly the opposite direction of the hierarchy in the production of these mesons, discussed above, that follows on HQET general grounds. BT quark models satisfy heavy quark scaling for the decay constants, namely $\sqrt{M} f=$ Const. and the relation $f_{B}=f_{B^{*}}$, together with the selection rule $f_{3 / 2}=0$. For the allowed decay constants $f_{1 / 2}$ one finds for the $n=0$ states, within 
the Godfrey-Isgur spectroscopic model [1] $\left.{ }_{1}^{1} \overline{1}\right]$ : $\sqrt{M} f_{1 / 2}^{(0)}=0.64 \mathrm{GeV}^{3 / 2}$, very close to the value found for the ground state $S$-wave $\sqrt{M} f^{(0)}=0.67 \mathrm{GeV}^{3 / 2}$ ( $M$ is the mass of the heavy meson). Therefore, assuming factorization, one predicts relations of the kind $\Gamma\left(\bar{B}_{d} \rightarrow D_{s_{0}}^{-}\left(\frac{1}{2}\right) D^{+}\right) / \Gamma\left(\bar{B}_{d} \rightarrow D_{s}^{-} D^{+}\right) \cong 1$.

In conclusion, we have underlined that there is at present a problem between the prediction in heavy quark limit of QCD ( rates is important, as they are directly related to the slope of the elastic IW function $\rho^{2}$. On the other hand, we have shown that Uraltsev SR is satisfied by Bakamjian-Thomas relativistic quark models, that provide a physical interpretation of the sum rule as due to the light spectator quark Wigner rotations, and not to a possible $\mathbf{L} \cdot \mathbf{S}$ force. Uraltsev Sum Rule yields a rationale for the bound $\rho^{2} \geq \frac{3}{4}$ found within the BT quark models some years ago. On the other hand, the heavy quark limit selection rule $f_{3 / 2}^{(n)}=0$, plus the factorization hypothesis predicts an opposite hierarchy for the emission of $P$-wave mesons.

The authors acknowledge partial support from TMR-EC Contract No. CT98-0169.

\section{References}

[1] N. Uraltsev, Phys. Lett. B $501-1001) 8 \overline{6}_{1}^{1} ;$ hep-ph/0012336.

[2] J. D. Bjorken, invited talk at Les Rencontres de Physique de la Vallée d'Aoste, La Thuile, SLAC-PUB-5278, 1990 ; N. Isgur and M. B. Wise, 'P hys. Rev. D $43-1991) 819$ '.

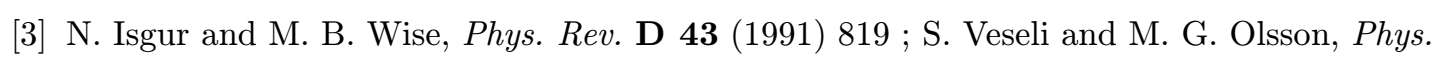
Rev. D $54(19 \overline{9} 6)-8 \overline{8}$.

[4] B. Bakamjian and L. H. Thomas, Phys. Rev. 92 (1953) 1300 ; A. Le Yaouanc, L. Oliver, O. Pène and J.-C. Raynal, Phys. Lett. B_365 1996$) 319$.

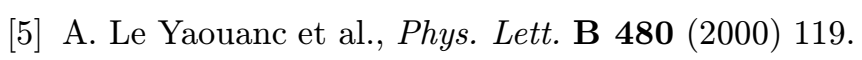

[6] A. F. Falk and M. Neubert, 'Phys. Rev. D 47

[7] M. Voloshin,

[8] M. Neubert, 'Phys. Rev. D $\mathbf{4} 6(1992) 1076$

[9] A. Le Yaouanc, V. Morénas, L. Oliver, O. Pène and J.-C. Raynal, hep-ph/0105247.

[10] M. Beneke, G. Buchalla, M. Neubert and C. T. Sacharajda, iNucl. Phys. B 591

[11] M. Bataglia, hep-ex/0009026, Experimental Status of Semileptonic B Decays.

[12] CLEO CONF 99-6, hep-ex/09908009.

[13] A. Le Yaouanc, L. Oliver, O. Pène and J.-C. Raynal, IPhys. Lett. B $408 \overline{0}(1997)$

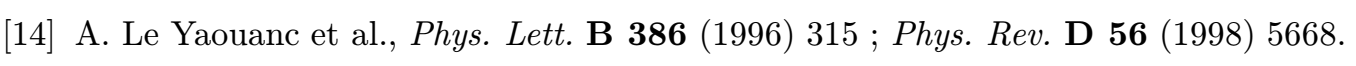

[15] A. Le Yaouanc et al., Phys. Rev. D 58 (1998) 114019.

[16] S. Godfrey and N. Isgur, iPhys. Rev. D' $32-1985)$ 189."

[17] P. Cea, P. Colangelo, L. Cosmai and G. Nardulli, 'Phhys. Lett. B $206-198886911$.

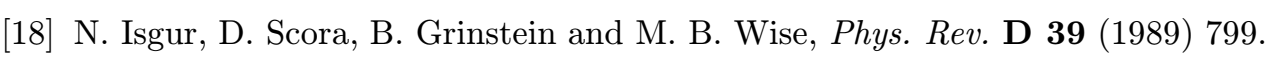

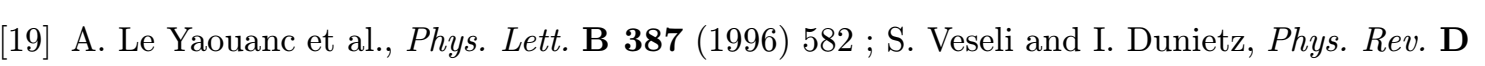
- - 54 (1996) 6803 